Original Article

\title{
The effects of gait time and trunk acceleration ratio during stair climbing in old-old adult females
}

\author{
Sun-SHIL SHIN ${ }^{1)}$, Won-GYu YoO ${ }^{2}{ }^{*}$ \\ 1) Department of Rehabilitation Science, The Graduate School, Inje University, Republic of Korea \\ 2) Department of Physical Therapy, College of Biomedical Science and Engineering, Inje University: \\ 607 Obangdong, Gimhae, Gyeongsangnam-do 621-749, Republic of Korea
}

\begin{abstract}
Purpose] This study investigated the effects of gait time and trunk acceleration ratio in old-old adult females during stair climbing. [Subjects and Methods] Twenty-five older adult females who were able to walk independently volunteered for this study and were categorized into two age groups (older adults or old-old adults). Gait time and trunk acceleration ratio were measured using an accelerometer during stair climbing. [Results] Gait time and trunk acceleration ratio when climbing stairs were significantly higher in the old-old age group than in the older adults group. [Conclusions] These findings suggest that old-old females have decreased upper trunk control. In addition, gait time and the trunk acceleration ratio during stair climbing are useful clinical markers for predicting function and balance control ability in old-old elderly populations.

Key words: Center of mass acceleration, Old-old elderly, Turning gait
\end{abstract}

(This article was submitted Jan. 29, 2016, and was accepted Apr. 7, 2016)

\section{INTRODUCTION}

Ascending stairs is a common activity of daily living that is essential for independence and quality of life ${ }^{1)}$. Safe stair climbing demands adequate muscle strength to propel the body up the stairs. In addition, during stair negotiation, trunk movements are important with respect to postural control, since the trunk supports the head and lower extremities by regulating both the amplitude and structure of gait-related oscillations and provides input to the visual and vestibular sensory systems ${ }^{1)}$. Therefore, age-related musculoskeletal changes lead to muscle strength decline and limit both balance and gait ${ }^{2}$. To date, self-report questionnaires have been used to assess stair negotiation as a part of functional assessments of older adults. Recent research suggests some limitations of using self-report questionnaires to assess stair climbing. For example, the questionnaires cannot evaluate the quality of the movements, which is important because difficulty in executing such movements may be a reliable and valid indicator of stair climbing impairments ${ }^{3}$. Oh-Park et al. suggest that the time needed to negotiate three steps is a meaningful measure in clinical settings because the risk of functional deterioration in older adults can be easily assessed by this method ${ }^{4}$. In addition, the trunk acceleration ratio has been widely used to assess dynamic balance in older adults ${ }^{1}$. Therefore, the purpose of this study was to investigate the stair ascending time and trunk acceleration ratio during stair climbing in old-old adult females.

\section{SUBJECTS AND METHODS}

A total of 25 older females volunteered for this study and were categorized into two age groups: older adults (65-74 years) and old-old adults ( $\geq 75$ years). There were a total of 13 adults in the older adults group, with an average age, weight, and

\footnotetext{
*Corresponding author. Won-Gyu Yoo (E-mail: won7y@inje.ac.kr)

(C)2016 The Society of Physical Therapy Science. Published by IPEC Inc.

This is an open-access article distributed under the terms of the Creative Commons Attribution Non-Commercial No Derivatives (by-nc-nd) License $<$ http://creativecommons.org/licenses/by-nc-nd/4.0/>.
} 
height of $71.8 \pm 2.7$ (mean $\pm \mathrm{SD}$ ) years, $51.1 \pm 6.4 \mathrm{~kg}$, and $151.5 \pm 3.4 \mathrm{~cm}$, respectively. The old-old adults group comprised 12 adults with an average age, weight, and height of $80.4 \pm 4.6$ years, $49.0 \pm 6.7 \mathrm{~kg}$, and $148.9 \pm 3.5 \mathrm{~cm}$, respectively. All participants were able to walk independently without the use of any assistive device and all had a score greater than 24 on the Korean Version of the Mini-Mental State Exam. Approval for this study was obtained from the Inje University Ethics Committee for Human Investigations, and written informed consent was obtained from all participants. Gait time and trunk acceleration ratio during stair climbing were measured with a tri-axial accelerometer (Fit Dot Life, Suwon, Korea). The gait time was calculated according to accelerometer data ${ }^{5}$. Trunk acceleration was calculated by obtaining the vector sums of the anterior-posterior, medial-lateral, and vertical acceleration amplitudes. The trunk acceleration ratio was then calculated by dividing the T2 trunk acceleration value by the $\mathrm{L} 3$ trunk acceleration value ${ }^{1)}$. We used three 3 -step staircases (rise $=17 \mathrm{~cm}$, tread $=28 \mathrm{~cm}$, width $=50 \mathrm{~cm}$ ) placed directly in front of a larger staircase ${ }^{4)}$. Each participant was asked to ascend the three stairs at their own pace, to use the dominant foot for the first step, and to place only one foot on each step (i.e., to progress in a "step-over" manner). The differences in gait times and trunk acceleration were analyzed using independent t-tests. The SPSS for Windows statistical software package (ver. 18.0; SPSS Inc., Chicago, IL, USA) was used for the statistical analysis. The level of statistical significance was set at $\mathrm{p}<0.05$.

\section{RESULTS}

Gait time and the trunk acceleration ratio when climbing stairs were significantly higher in the old-old age group (4.1 \pm $0.8 \mathrm{~s}$ and $2.2 \pm 0.6$, respectively) than in the older adults group $(3.5 \pm 0.4 \mathrm{~s}$ and $1.6 \pm 0.6$, respectively; $\mathrm{p}<0.05)$.

\section{DISCUSSION}

Gait time and trunk acceleration ratio during stair climbing in old-old adult females were assessed in this study. Our findings indicated that although there was no difference between the groups in terms of lower extremity muscle strength, functional and trunk balance control ability during stair climbing decreased in the old-old adult group compared to the older adult group. Oh-Park et al. investigated the predictive utility, in terms of functional decline, of stair negotiation times ${ }^{4}$. They reported that the 3-stair negotiation time was a meaningful measurement in a clinical setting that allowed the risk of functional decline in older adults, including high-functioning individuals, to be easily assessed. In addition, they determined the stair negotiation time to be $0.5 \mathrm{~s}$ for significant deterioration and $0.2 \mathrm{~s}$ for significant improvement. The trunk acceleration ratio serves as both an index of trunk oscillations and a predictor of falling ${ }^{1,5}$. Trunk acceleration is defined as the rate of change of trunk velocity, and a higher trunk acceleration ratio indicates that the velocity of the upper trunk changes frequently during stair negotiation. Our results also indicated that old-old females had decreased upper trunk control, which minimized the motion of the head and increased the probability of falling. Therefore, we suggest that ascending stairs is one of the biggest balance challenges in old-old adults and that gait time and the trunk acceleration ratio are useful clinical markers for predicting falls in old-old populations.

\section{REFERENCES}

1) Doi T, Hirata S, Ono R, et al.: The harmonic ratio of trunk acceleration predicts falling among older people: results of a 1-year prospective study. J Neuroeng Rehabil, 2013, 10: 7. [Medline] [CrossRef]

2) Cabell L, Pienkowski D, Shapiro R, et al.: Effect of age and activity level on lower extremity gait dynamics: an introductory study. J Strength Cond Res, 2013, 27: 1503-1510. [Medline] [CrossRef]

3) Verghese J, Wang C, Xue X, et al.: Self-reported difficulty in climbing up or down stairs in nondisabled elderly. Arch Phys Med Rehabil, 2008 , 89: 100-104. [Medline] [CrossRef]

4) Oh-Park M, Wang C, Verghese J: Stair negotiation time in community-dwelling older adults: normative values and association with functional decline. Arch Phys Med Rehabil, 2011, 92: 2006-2011. [Medline] [CrossRef]

5) Shin SS, Yoo WG: Effects of gait velocity and center of mass acceleration during turning gait in old-old elderly women. J Phys Ther Sci, 2015, 27: 1779-1780. [Medline] [CrossRef] 